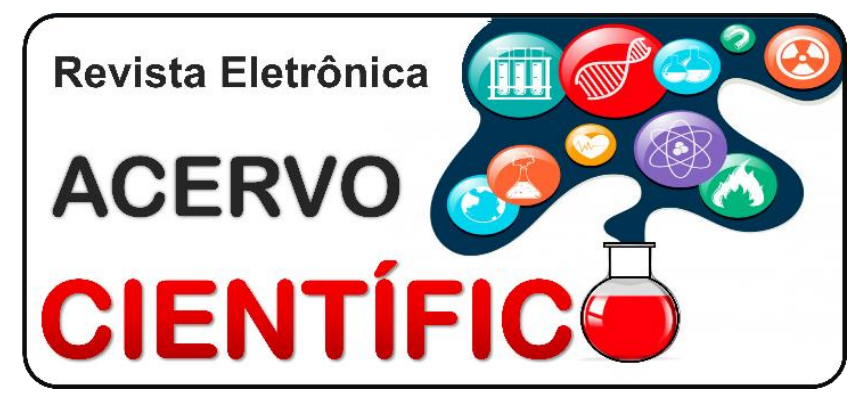

REVISÃO BIBLIOGRÁFICA

Recebido em: 7/2020

Aceito em: 8/2020

Publicado em: 11/2020

\title{
Impactos biológicos e sociais na vida das mulheres com Síndrome dos Ovários Policísticos
}

\author{
Physical and social impacts on the life of women with Polycystic Ovary Syndrome
}

Impactos físicos y sociales en la vida de las mujeres con Síndrome del Ovario Poliquistico

Isabella Ferraz Ferreira ${ }^{1 *}$, Danielle Fiorin Ferrari Novais², Elder Costa Fernandes ${ }^{3}$, Fernanda de Almeida Ferman Vieira ${ }^{1}$, Jéssica Vieira Barros ${ }^{3}$, Lara Martins da Silva ${ }^{1}$, Larissa de Sá Abdu${ }^{1}$, Marcella Moreira Souza Andrade ${ }^{3}$, Roberta Nantes Costa ${ }^{3}$, Thainah Pereira Rocha ${ }^{3}$.

Resumo: Este artigo buscou enfatizar a importância de uma abordagem precoce da SOP nos diversos setores da medicina. Concomitantemente, expôs o quadro clínico das pacientes afetadas, nos âmbitos psicológico, endócrino e dermatológico. Pode-se destacar como principais mudanças estéticas a acne, o hirsutismo, a dermatite seborréica, a alopecia androgênica, a acanthosis nigricans e a obesidade, todas influenciadas por fatores genéticos e ambientais. Além disso, a infertilidade também é uma consequência agravante para quadros depressivos. Para essas manifestações, respectivamente, a isotretinoína, o anticoncepcional combinado, mudanças dos hábitos de vida e a metformina devem ser utilizados para minimizar os efeitos deixados pela síndrome, podendo, ou não, serem usados em associação, conforme a necessidade individual de cada paciente. Nesta revisão bibliográfica, concluiu-se que um tratamento prévio e multidisciplinar evita fármacos desnecessários e transtornos psicológicos (estresse, ansiedade, depressão e quadros psicóticos), advindos das sequelas deixadas por esta doença, quando não há o acompanhamento conveniente.

Palavras-chave: Síndrome do ovário policístico, Manifestações cutâneas, Hiperandrogenismo.

Abstract This article quests to emphasize the importance of an early treatment for PCOS in the different sectors of medicine. Concomitantly, he exposed the clinical of the affected patients, in the psychological, endocrine and dermatological scope. Acne, hirsutism, seborrheic dermatitis, androgenic alopecia, acanthosis nigricans and obesity can all be highlighted as main aesthetic changes, all influenced by genetic and environmental factors. In addition, infertility is also an aggravating consequence for depressive conditions. For these manifestations, respectively, isotretinoin, combined contraceptives, changes in lifestyle and metformin should be used to minimize the effects left by the syndrome, and may, or may not, be used in combination, according to the individual need of each patient. In this bibliographic review, we conclude that a previous and multidisciplinary treatment avoids unnecessary drugs and psychological disorders (stress, anxiety, depression and psychotic conditions), resulting from the sequelae left by this disease, when there isn't the timely monitoring.

Keywords: Polycystic ovary syndrome, Cutaneous manifestations, Hyperandrogenism.

Resumen: Este artículo busca enfatizar la importancia de un enfoque temprano para SOP en los diferentes sectores de la medicina. Concomitantemente, expuso el cuadro clínico de los pacientes afectados, en el ámbito psicológico, endocrino y dermatológico. Los principales cambios estéticos a destacar son el acné, el hirsutismo, la dermatitis seborreica, la alopecia androgénica, la acantosis nigricans y la obesidad, todos influenciados por factores genéticos y ambientales. Además, la infertilidad también es una consecuencia agravante para las condiciones depresivas. Para estas manifestaciones, respectivamente, la isotretinoína, los anticonceptivos combinados, los cambios en el estilo de vida y la metformina deben usarse para minimizar los efectos que deja el síndrome, y pueden usarse o no en combinación, de acuerdo con las necesidades individuales de cada paciente. En esta revisión bibliográfica, se concluyó que un tratamiento

\footnotetext{
${ }^{1}$ Universidade de Vassouras (UV), Vassouras - RJ. *E-mail: ferrazisabella@hotmail.com

2 Faculdade Multivix, Vitória - ES.

${ }^{3}$ Centro Universitário de Caratinga (UNEC), Caratinga - MG.
} 
previo y multidisciplinario evita las drogas innecesarias y los trastornos psicológicos (estrés, ansiedad, depresión y condiciones psicóticas), como resultado de las secuelas dejadas por esta enfermedad, cuando no hay un monitoreo conveniente.

Palabras clave: Síndrome del ovario poliquístico, Manifestaciones cutáneas, Hiperandrogenismo.

\section{INTRODUÇÃO}

Considerada uma das endocrinopatias mais comuns do sexo feminino, a Síndrome do Ovário Policístico (SOP) é uma doença multifatorial que acomete 5 a $10 \%$ das mulheres em idade reprodutiva. Devido à interação de fatores genéticos e ambientais, que se combinam e contribuem para fisiopatologia e clínica, a SOP é um distúrbio de alta complexidade (KEEN MA, et al., 2017; ROJAS PMJ e FERNANDEZ MEK, 2018).

Segundo Housman E e Reynolds VA (2014), trata-se de uma condição hiperandrogênica, muito relacionada à obesidade e à resistência insulínica, que se expressa através de alterações metabólicas, ovulatórias e sinais cutâneos. Fato esse que faz a SOP frequentemente ser diagnosticada em uma consulta dermatológica. Seu diagnóstico tem como base a presença de, pelo menos, dois dos três critérios de Rotterdam (2003). Neles estão inclusos o hiperandrogenismo (clínico ou bioquímico), oligomenorréia ou amenorréia, e ovários policísticos ao ultrassom (presença de 12 ou mais folículos em cada ovário medindo entre 2 e $9 \mathrm{~mm}$ ou volume ovariano maior que $10 \mathrm{~cm}^{3}$ ) (KEEN MA, et al., 2017).

A repercussão clínica desta doença promove mudanças significativas na qualidade de vida da paciente, tanto no âmbito biológico, quanto no âmbito social. Manifestações como irregularidade menstrual e infertilidade, decorrentes do distúrbio da ovulação, são queixas frequentes nos consultórios ginecológicos. Além disso, a SOP está associada a distúrbios metabólicos, predispondo ao Diabetes mellitus tipo 2, dislipidemia e aumento de doenças cardiovasculares. Todos esses distúrbios estão relacionados ao hiperandrogenismo e à resistência insulínica, presentes na síndrome, que vão se manifestar também de forma cutânea (LAZARIDOU S, et al., 2017; GUNNING MN e FAUSER MJC 2017; HOUSMAN E e REYNOLDS VA 2014; PASCH L, et al., 2016).

Existe uma gama de manifestações dermatológicas na SOP que impactam negativamente na autoestima e nas relações sociais das pacientes que as apresentam, como acne, hirsutismo, dermatite seborreica, acanthosis nigricans e alopecia. A gravidade dos sintomas costuma ser proporcional ao nível de insatisfação e constrangimento das pacientes em relação a sua aparência física perante à sociedade, o que resulta, muitas vezes, em afastamento social. A acne e o hirsutismo, considerados os principais fatores associados ao estresse emocional e social, juntamente com a infertilidade, geram consequências desfavoráveis ao bemestar das pacientes afetadas, aumentando a incidência de depressão (MOREIRA S, et al., 2013).

O objetivo deste artigo consiste em abordar a importância de um acompanhamento multidisciplinar frente a essa patologia multifatorial. Dessa forma, a participação de médicos ginecologistas, dermatologistas, cardiologistas e, quando necessário, psicólogos, são imprescindíveis, e visam, em conjunto, minimizar as sequelas físicas e psicológicas das mulheres afetadas, além de melhorar a qualidade de vida dessas pacientes.

\section{REVISÃO BIBLIOGRÁFICA}

\section{Definição e Fisiopatologia}

A síndrome dos ovários policísticos (SOP) é uma endocrinopatia muito comum em mulheres em idade reprodutiva. Descrita em primeiro momento como Síndrome de Stein-Leventhal, foi descoberta em 1935, pelos médicos Irving Stein e Michael Leventhal, através de um procedimento cirúrgico que constatou a presença de múltiplos cistos nos ovários, em pacientes que estavam em estado de anovulação (HOUSMAN E e REYNOLDS VA, 2014).

Posteriormente, a síndrome passou a ser nomeada como SOP, para associar o nome à morfologia dos ovários e, assim, caracterizar melhor a doença. A patologia provoca estados de hiperandrogenismo, 
anovulação crônica e alteração ultrassonográfica de ovários policísticos. Essas alterações estão relacionadas à sua fisiopatologia, que embora não esteja amplamente confirmada, há consensos que afirmam tratar-se de uma doença multifatorial provocada pela interação de fatores genéticos, ambientais e, até mesmo, intrauterinos (HOUSMAN E e REYNOLDS VA, 2014; MOLINA MTC, et al., 2015; ROJAS JM e FERNANDEZ MEK, 2018; SPRITZER PM, 2013).

Os efeitos genéticos são passados por gerações e são responsáveis por tornar os pacientes candidatos à resistência insulínica na puberdade e à produção de androgênio. Esses efeitos são encontrados na SOP e também se relacionam com os fatores ambientais e, assim, um paciente com sobrepeso pode aumentar sua predisposição à doença. Outro influenciador é a etnia, uma vez que o hirsutismo presente na patologia é menos frequente em pacientes asiáticos (SPRITZER PM, 2013; HOUSMAN E e REYNOLDS VA, 2014).

Já os efeitos intra-uterinos são associados à exposição a glicocorticóides e andrógenos durante a formação fetal. Foi observado que essa exposição pode alterar a expressão gênica, aumentando o risco de distúrbios metabólicos e reprodutivos na vida adulta, como os evidenciados nas pacientes afetadas pela patologia em questão. Caso isso ocorra no período neonatal, as mesmas alterações também poderão ser vistas. A combinação desses fatores leva à patogênese da doença que inicia no comprometimento do processo de maturação folicular e de ovulação. Ocorre uma disfunção na enzima CYP17 alfa e no citocromo, alterando a produção de androgênios e acarretando uma atresia folicular prematura, seguida de anovulação (MOLINA MTC, et al., 2015; HOUSMAN E e REYNOLDS VA, 2014).

A taxa de estrogênio é alterada e tem como consequência o aumento na produção de hormônio liberador de gonadotrofina $(\mathrm{GnRH})$ pelo hipotálamo. Esse hormônio também é estimulado devido aos baixos níveis de progesterona, que não são modificados na fase lútea. Tal fato aciona a glândula pituitária anterior a ter uma preferência na produção do hormônio luteinizante (LH) sobre o hormônio folículo estimulante (FSH) (MOLINA MTC, et al., 2015; HOUSMAN E e REYNOLDS VA, 2014). Como o FSH encontra-se baixo, a maturação folicular é inibida, resultando em um folículo dominante, que não se sobressai, e na manutenção de folículos em estágios iniciais. Dessa forma, o aspecto policístico e a anovulação crônica são configurados.

Já o LH, cuja produção é estimulada, promove hiperplasia das células tecais e leva ao aumento na produção de androgênios. Além disso, existe síntese de androstenediona, que pode ser convertida em testosterona ou aromatizada e convertida em estrogênio. Caso a produção de estrogênio seja ampliada, esse atuará na hipófise, inibindo mais a secreção de FSH e estimulando mais a de $\mathrm{LH}$, perpetuando o ciclo. A proliferação e diferenciação do endométrio também são estimulados e, uma vez que não há atuação da progesterona, o risco de hiperplasia e câncer endometrial se eleva (ROJAS JM e FERNANDEZ MEK, 2018; HOUSMAN E e REYNOLDS VA, 2014).

Assim como o LH, a insulina desempenha seu papel na SOP, pois ela estimula a célula da teca ovariana na secreção de andrógenos. Esse hormônio também atua na testosterona livre que é elevada pela inibição da produção hepática de hormônio sexual e globulina de ligação (SHBG). Pacientes obesas que apresentam resistência insulínica, normalmente aumentam a secreção pancreática, gerando um círculo vicioso, devido ao efeito anabólico do excesso de andrógenos e da insulina, que, consequentemente, leva a um maior grau da obesidade (MOLINA MTC, et al., 2015; ROJAS JM e FERNANDEZ MEK, 2018; HOUSMAN E e REYNOLDS VA, 2014; RIBEIRO BV, et al., 2019).

Outro fato observado é a influência das glândulas suprarrenais, pois assim como os ovários, essas produzem andrógenos sob influência dos hormônios liberados pelo hipotálamo e hipófise. E além de produzílos, impulsiona a secreção de precursores para esses andrógenos na circulação. Assim, pacientes com patologias envolvendo as suprarrenais, como hiperplasia adrenal congênita, apresentam maior incidência de SOP, dado que o aumento na produção de desidroepiandrosterona (DHEA) e sulfato de desidroepiandrosterona (DHEAS), andrógenos liberados por essas glândulas, levam ao semelhante quadro de hiperandrogenismo visto nos ovários policísticos. À partir disso, tem-se os efeitos locais, no desenvolvimento folicular ovariano, e sistêmicos que influenciam nas manifestações da doença (ROJAS JM e FERNANDEZ MEK, 2018). 


\section{Manifestações clínicas e diagnóstico}

A disfunção anovulatória e o hiperandrogenismo, características marcantes da SOP, desencadeiam uma série de manifestações clínicas. Sendo assim, os sinais e sintomas decorrem do acometimento de diversos sistemas, podendo ser cutâneos (hirsutismo, acne e alopecia), ginecológicos (oligomenorreia, amenorreia, ciclos menstruais irregulares) e metabólicos (obesidade, hipertensão, dislipidemia). Os sintomas podem variar de acordo com a etapa de vida da paciente, sendo mais evidentes pós-puberal. Nesse período, se sobrepõe a irregularidade menstrual, acne e hirsutismo. Com o envelhecimento, esses sintomas tendem a melhorar e a infertilidade passa a se tornar mais comum (SPRITZER PM, 2013; GUNNING MN e FAUSER MJC 2017).

Um estudo realizado em 100 mulheres diagnosticadas com SOP concluiu que 65\% apresentaram distúrbios menstruais. A alteração menstrual mais comumente encontrada foi oligomenorreia, seguida de amenorreia. Quanto às alterações metabólicas, a obesidade é uma característica muito comum nessas pacientes, sendo constatado, através do cálculo do IMC, a prevalência de $27 \%$ de obesidade e $53 \%$ de sobrepeso nas participantes da amostra (SPRITZER PM, 2013; KEEN MA, et al., 2017; TABARES RG, et al., 2018).

Ademais, essas mulheres ainda podem apresentar outras complicações metabólicas, como resistência insulínica, dislipidemia, hipertensão e síndrome metabólica, além de um maior risco de doenças cardiovasculares. De acordo com Housman E e Reynolds VA (2014), não está definido se a SOP é um fator de risco independente ou se é um desfecho das condições associadas à síndrome. Porém, em 2017, um estudo realizado por Oliveira FR analisou que a síndrome metabólica causa o aumento de sete vezes o risco cardiovascular nas portadoras de SOP. Outras consequências descritas foram a infertilidade e o câncer de endométrio. Ainda, devido à anovulação crônica, as pacientes apresentam ao longo do tempo maior chance dessas repercussões (HOUSMAN E e REYNOLDS VA, 2014; KEEN MA, et al., 2017).

O diagnóstico desta condição é dado pelos Critérios de Rotterdam de 2003, no qual, a presença de duas, das seguintes alterações: oligomenorreia ou anovulação, sinais bioquímicos ou clínicos de hiperandrogenismo e ovários policísticos ecográficos, confirma a síndrome (OLIVEIRA FR, et al., 2016).

\section{Manifestações dermatológicas}

As manifestações cutâneas mais comuns da SOP relacionadas ao hiperandrogenismo são acne, hirsutismo, dermatite seborreica (DS) e alopecia androgênica (AGA). Os traços de androgênio aumentado são mais presentes durante a puberdade, entretanto, podem aparecer após essa fase, principalmente na existência de um cenário de obesidade. Além dessas lesões, pacientes portadoras dessa síndrome podem apresentar também um sinal dermatológico referente à resistência insulínica, fator importante na fisiopatologia da doença, denominado acanthosis nigricans (AN) (SEKHON AK, et al.,2020; HOUSMAN E e REYNOLDS VA, 2014; HONG JS, et al., 2015).

A acanthosis nigricans apresenta-se normalmente na região cervical, com placas hiperpigmentadas aveludadas, mas também pode ser encontrada em regiões de axilas e virilhas. A insulina em nível aumentado se liga aos receptores de IGF-1 (fator de crescimento semelhante à insulina tipo 1) e estimula a proliferação de queratinócitos epidérmicos e fibroblastos dérmicos, promovendo o surgimento da AN. É observado, ainda, que a AN representa um fenótipo grave da SOP, como um sinal previsível dos riscos de doença cardiovascular, e tem uma prevalência de $30 \%$ nas mulheres com essa doença (HOUSMAN E e REYNOLDS VA 2014; ÁVILA MAPD, et al., 2014; KEEN MA, 2017; FENG J, et al., 2017).

Mais prevalente do que a AN, a acne aparece em $48 \%$ dessas pacientes. Uma apresentação de forma persistente na vida adulta, de aspecto inflamatório ou de início tardio, é marcada por comedões que evoluem para acne nodular e se distribuem normalmente na face inferior, costas, pescoço e tórax. Ademais, costuma não responder ou ter uma ínfima resposta à terapia usual e uma recidiva logo após a descontinuação do tratamento. Estrógenos, progesterona, insulina e IGF-1 são componentes patogênicos, assim como genética e dieta. Os hormônios androgênios agem aumentando a queratose folicular e a produção de sebo, componentes da patogenia da acne e da seborreia (KEEN MA, 2017; CORRALES MAM, 2016; FRANIK G, et al., 2018). 
A dermatite seborreica tem uma prevalência de $29 \%$ em pacientes com SOP. Além do hiperandrogenismo como um fator causal, é importante considerar também a herança genética do paciente, bem como o emocional e os fatores climáticos. Sendo assim, exames sorológicos, clínicos e radiológicos são indicados para confirmar se há relação entre a seborreia e a SOP. A alopecia androgênica, na mulher com SOP, tem prevalência de $31 \%$ e é marcada por uma perda de cabelo mais centralmente localizada no couro cabeludo, preservando a linha frontal. Dificilmente apresenta padrão masculino, com recessão frontotemporal e vértice. Como não é uma manifestação frequente, é dever do médico excluir outras causas, como anemia ferropriva, alterações na tireoide, entre outras condições (KEEN MA, 2017; HOUSMAN E e REYNOLDS VA, 2014).

Outro achado é o hirsutismo, manifestação que se correlaciona com uma alta produção de testosterona, e é marcado pelo crescimento excessivo de pelos grossos em uma distribuição masculina, normalmente presentes no lábio superior, aréola, queixo, tórax, costas e parte inferior do abdome. Está presente em até $60 \%$ das mulheres com SOP e seu grau oscila dependendo da etnia (SEKHON AK, et al.,2020; HOUSMAN E e REYNOLDS VA, 2014).

\section{Qualidade de vida e aspectos psicológicos}

Todas essas mudanças estéticas causam um impacto psicológico, que vem sendo um objeto de estudo cada vez mais importante, uma vez que aproximadamente 10\% dessas mulheres são acometidas por distúrbios psíquicos. Nota-se que as mulheres afetadas apresentam maior taxa de ansiedade, depressão, estresse, diminuição na qualidade de vida, alterações na imagem e identidade corporal, baixa autoestima e disfunção psicossexual. O hirsutismo é um grande responsável por esses sintomas, mas atesta-se que a obesidade é a condição que mais tem impacto na vida da paciente (HOUSMAN E e REYNOLDS VA, 2014; PASH L, et al., 2016; ACOSTA CAG, et al., 2015).

A baixo autoestima é um grande fator de risco para a depressão, considerada a doença psicológica que mais afeta as mulheres com SOP, principalmente, aquelas com IMC elevado. Além disso, a infertilidade é o desfecho mais relacionado à esta comorbidade. Porém, deve-se ressaltar, que mulheres com maior nível educacional e acesso à saúde têm menor prevalência de depressão, o que demonstra a importância de uma abordagem multidisciplinar de todos os pacientes de forma preventiva. Outra perturbação psicológica muito comum é a ansiedade, observada com maior prevalência nas obesas, mas ainda assim presente nas pacientes com IMC adequado. Esse distúrbio, que se deve à alta taxa de androgênios e à resistência insulínica, notadamente prejudica a relação da mulher com a família e com o trabalho (ACOSTA CAG, et al., 2015).

Analisa-se ainda, que mulheres com SOP tendem a ter características de personalidade neurótica e problemas para lidar e controlar as emoções, tal como a raiva. Essas situações deterioram ainda mais a qualidade de vida delas, que, também, pode ser afetada por outros fatores, como o diagnóstico tardio e a não observação de melhora com o tratamento adequado (ACOSTA CAG, et al., 2015).

\section{Tratamento e resposta do objetivo}

Haja vista, a SOP é uma síndrome complexa, que exige um tratamento adequado para diminuir o impacto no bem-estar das mulheres afetadas, sabe-se que existem consequências não apenas no aspecto físico, mas também no emocional. Para isso, é preciso uma equipe multidisciplinar que acompanhe a paciente ao longo de sua vida. Antes de optar por um tratamento específico, farmacológico ou não, é importante entender a paciente e suas possibilidades, individualizando-as, para que falhas sejam evitadas. Saber se há interesse em engravidar durante o tratamento é um dos pontos fundamentais para que haja adequação da conduta médica (MOREIRA SNT, et al., 2013).

A primeira medida a ser adotada é com relação a mudanças no estilo de vida. A redução significativa de peso, pode reduzir o excesso de androgênio e ajudar na irregularidade menstrual, além de diminuir a morbimortalidade e os estressores psicossociais. Acredita-se que a inclusão dessas mulheres em grupos terapêuticos, específicos para SOP, seja uma boa estratégia para um estímulo conjunto e adesão às mudanças propostas, como a práticas de exercícios físicos regulares e reeducação alimentar (MOREIRA SNT, et al., 2013; FREIRE A, et al., 2018). 
Em relação ao tratamento da acne nas portadoras de SOP, constatou-se que quando tratada com medicamentos usualmente utilizados em outras mulheres sem a síndrome, a resposta era insatisfatória. Além disso, quando interrompia-se seu uso, havia o retorno das lesões, reafirmando o caráter hormonal. Diante disso, faz-se necessário, para uma boa resposta ao tratamento, o uso de isotretinoína e do anticoncepcional combinado oral (ACO), que atua interferindo na atividade androgênica, melhorando também o hirsutismo. Dentro dos 3 primeiros meses, é possível observar resultados do uso de terapia com os ACOs para acne, já em relação ao hirsutismo, espera-se por mais de 6 meses (CORRALES MAM, 2016; MOREIRA SNT, et al., 2013). A depilação a laser também é uma opção para redução dos pelos, mostrando resultado positivo com as mulheres que fizeram esta opção, inclusive reduzindo seus sintomas de ansiedade e depressão (MOREIRA SNT, et al., 2013).

Sobre os efeitos antiandrogênicos da metformina, ainda não há um consenso. Porém, ela é amplamente utilizada em pacientes com SOP, atuando na sensibilização da insulina e na redução do índice de massa corporal. Além disso, é conhecido seu efeito na indução da ovulação, sendo interessante para aquelas que têm interesse em engravidar. Sua dose habitual para SOP é de 1500 a $2500 \mathrm{mg} / \mathrm{dia}$, com início gradual das doses (150 a $500 \mathrm{mg} / \mathrm{dia}$ ), até alcançar a dose terapêutica, com 1 a 2 meses, para minimizar seus efeitos colaterais (náuseas, desconforto abdominal, diarreia). Vale ressaltar que funções renais e hepáticas devem ser monitorizadas antes e durante o tratamento (MOLINA MTC, et al., 2015; CORRALES MAM, 2016; SPRITZER PM, 2013).

Como mencionado, as consequências da SOP aumentam sintomas depressivos e ansiosos, que somados às alterações corporais, impulsionam na necessidade de uma abordagem multidisciplinar, em torno de profissionais da área endocrinológica, dermatológica, ginecológica e psicológica. Dessa forma, os efeitos podem ser minimizados, deixando a mulher menos estigmatizada pela síndrome e mais satisfeita com seu corpo e sua autoestima. Deve sempre ser levado em consideração os sentimentos da paciente, para que o tratamento seja o mais adequado à rotina de vida e suas condições financeiras (MOREIRA SNT, et al., 2013).

\section{CONSIDERAÇÕES FINAIS}

Devido à alta incidência e caráter multifacetado dos sintomas da SOP, pode-se afirmar que é de notória necessidade que o diagnóstico da síndrome seja preciso e precoce, para assim evitar tratamentos farmacológicos indevidos e proporcionar uma melhora na qualidade de vida das mulheres acometidas. Ademais, é importante entender os sintomas em questão e associá-los corretamente a seus fatores desencadeadores para dessa forma guiar os exames e minimizar o grande sofrimento e constrangimento gerados pelas manifestações clínicas dessa enfermidade. Sendo assim, a divulgação científica das manifestações clínicas da SOP além de informar a população sobre uma condição tão frequente contribui para mitigar as preocupações das portadoras da síndrome.

\section{REFERÊNCIAS}

1. ACOSTA CAG, et al. El síndrome de ovario poliquístico: aspectos psicológicos. Rev Chil Obstet Ginecol., 2015; 80(4):341-347.

2. MAPD, et al. Acantose nigricante: inter-relações metabólicas inerentes à síndrome dos ovários policísticos. Rev Bras Ginecol Obstet., 2014; 36(9): 410-5.

3. CORRALES MAM. Acné de causas endocrinológicas. Medicina Legal de Costa Rica - Edición Virtual, 2016; 33(1).

4. FENG J, et al. Prevalence of dermatologic manifestations and metabolic biomarkers in women with polycystic ovary syndrom in north China. Journal of Cosmetic Dermatology, 2017; 1-7.

5. FRANIK G, et al. Hormonal and metabolic aspects of acne vulgaris in women with polycystic ovary syndrome. European Review for Medical and Pharmacological Sciences, 2018; 22: 4411-4418.

6. FREIRE A, et al. Síndrome de Ovario Poliquístico (SOP) en la adolescencia. Rev. Hosp. Niños (B. Aires), 2018; 60(270): 258-263.

7. GUNNING NM, FAUSER MJC. Are women polycystic ovary syndrome at increased cardiovascular disease risk later in life? Climacteric, 2017; 20:3. 
8. HONG JS, et al. Cutaneous manifestations of the subtypes of polycystic ovary syndrome in Korean patients. JEADV, $2015 ; 29: 42-47$.

9. HOUSMAN E, REYNOLDS VA. Polycistic ovary syndrome: A review for dermatologists, 2014; 847. e1-10.

10. KEEN MA, et al. Cutaneous Manifestations of Polycystic Ovary Syndrome: A Cross-Sectional Clinical Study, 2017; 8:104.

11. LAZARIDOU S, et al. Prevalence, pathogenesis and management of prediabetes and type 2 diabetes mellitus in patients with polycystic ovary syndrome. HORMONES, 2017; 16 (4): 373-380.

12. MOLINA CTM, et al. Actualización dele Síndrome de Ovário Poliquístico y su importancia en dermatologia. Rev. Chilena Dermatol., 2015; 31 (4): 401-409

13. MOREIRA S, et al. Qualidade de vida e aspectos psicossociais da sindrome dos ovários policisticos: um estudo qualiquantitativo. Rev Bras Ginecol Obstet., 2013; 35(11):503-10

14. OLIVEIRA FR, et al. Associação entre produto de acumulação lipídica e hirsutismo em pacientes com síndrome do ovário policístico, Rev Bras Ginec Obst., 2016; 38: 71-76

15. PASH, et al. Clinician vc Self-ratings of Hirsutism in Patients With Polycystic Ovarian Syndrome: Associations with quality of life and depression. JAMA Dermatology, 2016; e1-6.

16. RIBEIRO BV, et al. Association of measures of central fat accumulation indices with body fat distribution and metabolic, hormonal, and inflammatory parameters in women with polycystic ovary syndrome. Arch Endocrinol Metab., 2019; 63 (4).

17. ROJAS PMJ, FERNANDEZ MEK. Síndrome de Ovários Poliquísticos. Medicina Legal de Costa Rica - Edición Virtual, 2018; 35 (1).

18. SEKHON AK, et al. The Association Between Polycystic Ovary Syndrome and Its Dermatological Manifestations. CUREUS, 2020; 12(2): e6855.

19. SPRITZER MP, Polycystic ovary syndrome: reviewing diagnosis and management of metabolic disturbances. Arq Bras Endocrinol Metab., 2014; 58 (2): 182-187.

20. TABARES RG, et al. Hiperandrogenismo e distúrbios metabólicos em mulheres com síndrome dos ovários policísticos. Rev Cubana de Endocrinologia, 2018; 29 (3) 